\title{
Relación gobernaza y politícas de sostenibilidad en el sector bioenergético del Valle del Cauca*
}

\author{
Recibido: 10 de agosto de 2020 • Aprobado: 21 de septiembre de 2020 \\ https://doi.org/10.22395/seec.v23n55a3
}

\author{
María Fanny Castro Arbeláez ${ }^{* *}$ \\ Johyner Obregón Morales ${ }^{* * *}$
}

\section{RESUMEN}

El propósito de este trabajo es identificar la tipología de la gobernanza que ejerce la empresa líder de la cadena de valor del sector de la bioenergía del Valle del Cauca con sus proveedores, si el tipo de gobernanza es determinante en la transmisión de politicas de sostenibilidad. Para cumplir con este objetivo se hace un estudio de caso de cinco empresas líderes analizadas desde la perspectiva de la tipologia de gobernanza de (Gereffi, Humphrey y Sturgeon, 2005) a saber: Grupo Agroindustrial Riopaila Castilla; Manuelita, S.A.; Carvajal S.A. Pulpa y Papel; Gases de Occidente; y Smurfit Kappa Cartón de Colombia, S.A., las cuales son empresas relevantes y capaces de transferir políticas de sostenibilidad a sus proveedores. Los resultados indican que en la cadena de valor de este sector predominan los tipos de gobernanza cautiva y modular, lo que facilita la adopción de políticas de sostenibilidad por parte de los proveedores, pues estas empresas cuentan con políticas de sostenibilidad claramente definidas que se difunden a sus proveedores. Lo anterior indica que están dadas las condiciones para que se configure una cadena de valor sostenible.

\section{PALABRAS CLAVE}

Cadenas de valor sostenible; gobernanza; empresas líderes; proveedores; stakeholders; bioenergía.

\section{CLASIFICACIÓN JEL}

L25, L52, O13, O25, P28, Q42, O51

\section{CONTENIDO}

Introducción; 1. Tipología de la gobernanza de la cadena de valor; 2. Metodología; 3. Resultados; 4. Conclusiones; Bibliografía.

* Artículo de investigación derivado de la Tesis doctoral Modelo para la evaluación de desempeño de la cadena de valor sostenible del sector de la bionergia a partir de un enfoque de gobernanza de la empresa líder y sus proveedores, doctorado Gerencia Pública y Política Social, Universidad del Baja California, México. Grupo de investigación Armonización y Valuación Contable, Clasificado en C en la convocatoria de Colciencia; proyecto de investigación. Años 2018 - 2020.

** Contadora Pública, Universidad Antonio Nariño, Cali, Colombia. Especialista en Revisoría Fiscal, Universidad Santiago de Cali, Cali. Magíster en Administración de Empresas, Universidad del Valle, Cali, Colombia. Candidata a doctora en Gerencia Pública y Política Social, Universidad de Baja California, México. Docente de la Universidad Libre de Colombia, Cali, Colombia. Correo electrónico: mariaf.castroa@unilibre.edu.co. Orcid: 0000-0001-6451-3225

*** Economista, Universidad del Valle, Cali, Colombia. Magister en Economía Aplicada, Universidad el Valle. Docente Universidad Santiago de Cali, Cali, Colombia. Correo electrónico: johyner.obregon00@usc.edu.co. 


\title{
RELATION BETWEEN GOVERNANCE AND SUSTAINABILITY POLICIES IN THE BIO-ENERGETIC SECTOR OF VALLE DEL CAUCA
}

\begin{abstract}
The goal of this work is to identify the governance typology exercised by the leading company in the value chain of the bio-energetic sector in Valle del Cauca (Valley of the Cauca) with its suppliers if the type of governance is determinant in the transmission of sustainability policies. For fulfilling this goal, this research studies the case of five leading companies from the typology of the governance perspective (Gereffi, Humphrey y Sturgeon,2005), these are Riopaila Castilla Agro-Industrial Group Manuelita S.A.; Carvajal S.A. Pulpa y Papel (Pulp and Paper); Gases de Occidente, and Smurfit Kappa Cartón de Colombia S.A., which are relevant companies capable of transferring sustainability policies to their suppliers. The results show that in the value chain of this sector the modular and captive governance types are predominant, which facilitates the adoption of sustainability policies by the suppliers, given how these companies have clearly defined sustainability policies that are promulgated among their suppliers. This indicates that the conditions are met for configuring a sustainable value chain.
\end{abstract}

\section{KEY WORDS}

Sustainable value chain; governance; leading companies; suppliers; stakeholders; bio-energy.

\section{JEL CODES}

L25, L52, O13, O25, P28, Q42, Q51

\section{CONTENT}

Introduction; 1. Typology of the value chain governance; 2. Methodology; 3 . Results; 4. Conclusions; Bibliography.

\section{RELAÇÃO ENTRE GOVERNANÇA E POLÍTICAS DE SUSTENTABILIDADE NO SETOR DE BIOENERGIA DO VALLE DEL CAUCA*}

\section{RESUMO}

O objetivo deste trabalho é identificar a tipologia de governança exercida pela empresa líder na cadeia de valor do setor de bioenergia do Valle del Cauca com seus fornecedores, bem como saber se esse tipo de governança é decisivo na transmissão das políticas de sustentabilidade. Para cumprir esse objetivo, foi feito um estudo de caso de cinco empresas líderes, que foram analisadas sob a ótica da tipologia de governança (Gereffi, Humphrey e Sturgeon, 2005). As empresas que foram estudadas são: Grupo Agroindustrial Riopaila Castilla, Manuelita S.A., Carvajal S.A. Pulpa y Papel, Gases de Occidente e Smurfit Kappa Cartón de Colombia S.A. Essas empresas são relevantes e capazes de transferir políticas de sustentabilidade para seus fornecedores. Os resultados indicam que os tipos de governança cativa e modular têm predominância na cadeia de valor do setor, o que facilita que as políticas de sustentabilidade sejam adotadas pelos fornecedores, uma vez que essas empresas possuem políticas de sustentabilidade claramente definidas e disseminadas para seus fornecedores. Isso indica que existem condições para que uma cadeia de valor sustentável seja configurada.

\section{PALAVRAS-CHAVE}

Cadeias de valor sustentáveis; governança; empresas líderes; fornecedores; stakeholders; bioenergia.

\section{CLASSIFICAÇÃO JEL}

L25, L52, O13, O25, P28, Q42, Q51

\section{CONTEÚDO}

Introdução; 1. Tipologia de governança da cadeia de valor; 2. Metodologia; 3. Resultados; 4. Conclusões; Bibliografia. 


\section{INTRODUCCIÓN}

El relacionamiento de las empresas líderes con sus proveedores es un factor importante para la adopción de políticas de sostenibilidad en las cadenas de valor, ya que, los requerimientos realizados por las empresas líderes a los proveedores, en torno a las buenas prácticas en la dimensión ambiental, social y económica, se constituye en una alternativa para que estas políticas permeen a todas las empresas de la cadena. "Saber cómo está gobernada una cadena permite a los donantes y los profesionales del desarrollo determinar el tipo y la cantidad de asistencia que los compradores aportarían a sus proveedores para que estos mejoren" (Stancey y Garay, 2010 citado en Castro y Moreno, 2015). Por tanto, la gobernanza ejercida por las empresas líderes permite que las pequeñas y medianas empresas (Pymes) de la cadena adopten buenas prácticas de sostenibilidad. La gobernanza permite resaltar las prácticas concretas y formas organizacionales particulares bajo las cuales las empresas líderes y otros actores, toman decisiones (Gibbon et al citado por Pérez Ibáñez, 2019).

Es por eso por lo que el desempeño sostenible de las empresas líderes, desde un enfoque de gobernanza, debe integrar a los stakeholders que conforman la cadena de valor. Al respecto, en la teoría de las partes interesadas, Tamayo et al (2018) propone que las empresas deben prestar atención a los grupos o individuos que conforman el sector al que pertenece la empresa, pues estos pueden afectar el propósito de una organización e impedir que consiga sus logros. En lo que respecta al desempeño en materia de sostenibilidad, Seuring y Müller (2008) argumentan que las empresas líderes de las cadenas de valor pueden ser responsables del desempeño ambiental y social de sus proveedores. La explicación radica en que las compañías líderes son aquellas compañías que generalmente gobiernan la cadena de suministro, proporcionan el contacto directo al cliente y diseñan el producto o servicio ofrecido.

En este sentido las empresas líderes (Empresas Grandes)' son importantes en la implementación de políticas sostenibles y el impulso de estas en los diferentes eslabones de la cadena y, para ello, se requiere de un ejercicio de gobernanza. Entendida esta como la manera en que el poder es ejercido en la gestión de los recursos económicos, sociales y ambientales (Kardos, 2012), los cuales, en este caso, contribuyen a que la cadena de valor opere de manera sostenible y esto se traduzca también en una mejora en la competitividad de la misma.

El propósito de este trabajo es explicar el patrón de gobernanza de la cadena de valor del sector de la bioenergía del Valle del Cauca, y así determinar la posibilidad de transferencia de políticas de sostenibilidad de la empresa líder a sus proveedores. El

Empresas Grandes: aquellas que cuentan con más de 200 empleados y activos superiores a 30.000 SMLV. 
Valle de Cauca es uno de los departamentos más importantes de Colombia, siendo su principal actividad económica la agricultura, destacándose la siembra la caña de azúcar. El departamento es reconocido por la industria azucarera, la cual provee los mercados de Colombia y otros países.

Para ello, en la primera sección se toman como punto de referencia los trabajos realizados por Gereffi et al (2005) y Gereffi (2014) en donde el concepto de gobernanza explica las diferentes maneras que tienen las empresas para organizar su producción transfronteriza (Pérez Ibáñez, 2019, p.67); en el presente trabajo se utiliza el termino para explicar el patrón de gobernanza en las cadenas de valor, con el fin de conocer los determinantes claves para la gobernanza de la misma y, de esta manera, definir las tipologías de gobernanza y su incidencia en la adopción de politicas sostenibilidad en la cadena. En la segunda sección se presenta la metodología, la cual consiste en un estudio de caso en el que se analiza la tipología de las cinco empresas líderes del sector de la bioenergía del Valle del Cauca. En la tercera sección se muestran los resultados y, por último, en la cuarta se presentan las conclusiones de la investigación.

\section{TIPOLOGÍA DE LA GOBERNANZA EN LA CADENA DE VALOR}

Con dos estudios realizados en la industria electrónica y la fabricación por contrato, (Sturgeon, 2013), contrastó tres tipos de relaciones con los proveedores basado en el producto y en el proceso: 1.- Proveedor de productos básicos, relación de mercados independientes, 2.- Proveedor cautivo, fabrica productos de acuerdo a la necesidad del comprador, y 3.- El proveedor llave en mano, produce bienes personalizados a varios clientes. Este enfoque enfatiza la complejidad de la información y el grado de los activos de producción. Por su lado Humphrey y Schmitz (2002) distinguieron relaciones con proveedores y compradores cuasi-jerárquicas, situación que corresponde a "proveedores cautivos" y relaciones de red entre empresas que cooperan ya que tienen competencias complementarias, en este enfoque los autores enfatizan el papel de la competencia del proveedor en la determinación del grado de subordinación, proveedor-cliente (Gereffi et al, 2005).

Con los enfoques anteriores Gereffi et al (2005) proponen una tipología de la gobernanza de la cadena de valor, sustentadas en las diferentes teorías que explican la organización de la industria. Las relaciones basadas en el mercado entre empresas y empresas integradas verticalmente (jerárquica), conforman extremos opuestos de un espectro de coordinación explicita y las relaciones de red comprenden un modo intermedio de gobernanza de la cadena de valor, por tanto, los autores proponen de acuerdo a la categoría de red tres tipos más: modular, relacional y cautivo; así, 
la tipología identifica cinco tipos de gobernanza en la cadena de valor, derivados de observación empírica. Los cinco tipos propuestos por los autores son:

A. Mercados: vínculos con transacciones repetidas, aunque los costos de cambiar a nuevos proveedores son bajos para las dos partes.

B. Cadenas de valor modulares: los proveedores de estas cadenas elaboran productos según la descripción y requerimientos del cliente y se hacen responsables de la tecnología, la maquinaria y desembolso de capital para componentes y materiales; la información, codificabilidad y las capacidades de los proveedores son elevadas.

C. Cadenas de valor relacionales: en estas redes se evidencian interacciones complejas entre compradores y vendedores, creando dependencia mutua y altos niveles de especificidad de los bienes. Se gestiona básicamente a través de la reputación, lazos familiares o étnicos; "las cadenas relacionales suelen darse en aglomeraciones productivas tales como los distritos industriales, en los que se combinan proximidad geográfica y un sustrato histórico-culturalmoral común entre las partes" (Mitnik, 2011 citado en Santarcángelo et al, 2017). Las condiciones de relacionamiento en esta cadena requiere de tiempo y cambiar de proveedor o comprador ocasiona costos elevados.

D. Cadenas de valor cautivas: estas redes se destacan por que las transacciones se dan entre pequeños proveedores aliados dependiente de compradores mucho más grande, se caracteriza por un alto grado de acompañamiento y control por parte de las empresas líderes. Los proveedores enfrentan un costo alto si cambian de cliente, por lo que son "cautivos".

E. Jerárquicas: se caracteriza por la integración vertical, la forma de dominancia es el control de gestión entre la empresa matriz y sus subordinadas.

Estos cinco tipos se combinan con tres patrones clave de la gobernanza: complejidad de las transacciones; codificabilidad de la información y capacidad de los proveedores (Gereffi et al, 2005).

Cuando los productos son estandarizados, y existe una buena información de precio y especificaciones generales sobre el bien, se evidencia un estilo de gobernanza basada en el mercado; por tanto, las capacidades de coordinación entre proveedores y compradores son bajas. Se habla de cadenas modulares cuando la información, la codificación y las capacidades del proveedor son altas, los productos son elaborados por los proveedores de acuerdo a los requerimientos especificos del cliente y asumen la plena responsabilidad por la tecnología del proceso (Santarcángelo et al, 2017); la 
información fluye a través de vínculos firmes y las asimetrías de poder entre proveedor-cliente son reducidas, aun así, el costo de cambiar a nuevos socios sigue siendo bajo. Si, por otro lado, las especificaciones del producto no se pueden codificar, las transacciones son complejas y las capacidades del proveedor son altas, se puede decir que la gobernanza de la cadena es relacional, en este tipo, la información implícita que se intercambian entre compradores y vendedores es alta, por tanto, se crea dependencia y confianza entre las partes (Gereffi et al, 2005; Gereffi, 2014). En el tipo de gobernanza cautiva, el flujo de información es altamente complejo y codificable, pero a diferencia de los otros tipos los proveedores no tienen las mismas capacidades de sus clientes, en este caso los compradores dan las instrucciones explicitas a cerca de qué, cómo y cuándo producir y ejercen un monitoreo, por lo que se genera una asimetría alta; en ocasiones los clientes, con el objetivo de aumentar la capacidad de sus proveedores, invierten recursos para mejorar la relación calidad-precio de los insumos (Santarcángelo et al, 2017). Por último, cuando las especificaciones del producto no se pueden codificar y no se encuentran proveedores competentes, las empresas internalizan las actividades creando vínculos con empresas filiales o subsidiarias. Esta forma de gobernanza esta impulsada por la necesidad de intercambiar conocimiento, así como la obligación de administrar redes complejas de entradas y salidas para controlar recursos, especialmente de propiedad intelectual (Gereffi et al, 2005; Gereffi, 2014; Santarcángelo et al, 2017).

En la tabla 1 se aprecia la incidencia de los determinantes claves de la gobernanza con los tipos propuestos. Al aumentar la complejidad de las transacciones, reduce la competencia del proveedor con relación a nuevas demandas; al disminuir la complejidad, hay mayor facilidad de codificación; el grado por el cual la complejidad puede mitigarse mediante la codificación, y la medida en que los proveedores tiene las capacidades necesarias para cumplir con el requisito de los compradores.

Tabla 1. Determinantes clave de la gobernanza en la cadena de valor.

\begin{tabular}{ccccc}
\hline $\begin{array}{c}\text { Tipo de } \\
\text { Gobernanza }\end{array}$ & $\begin{array}{c}\text { Complejidad de } \\
\text { las transacciones }\end{array}$ & $\begin{array}{c}\text { Capacidad para } \\
\text { codificar transacciones }\end{array}$ & $\begin{array}{c}\text { Capacidades en la } \\
\text { base de suministros }\end{array}$ & $\begin{array}{c}\text { Grado de coordinación explicita } \\
\text { y asimetría de potencia }\end{array}$ \\
\hline Marcado & Baja & Alta & Alta & Baja \\
Modular & Alta & Alta & Alta \\
Relacional & Alta & Baja & Alta \\
Cautivo & Alta & Alta & Baja \\
Jerárquica & Alta & Baja & Baja & $\downarrow$ \\
& & & & Alta \\
\hline
\end{tabular}

Fuente: Gereffit Humphrey y Sturgeon (2005) 
En la figura 1 se muestra claramente los cinco tipos de gobernanza, asociados a la coordinación explicita y la asimetria de poder.

Las lineas delgadas representan el intercambio basado en el precio, en tanto las flechas más anchas representan flujo de información y control, la gobernanza basada en el mercado demuestra que tanto coordinación explicita y asimetría de poder es baja; el extremo opuesto, coordinación y asimetría alta, se refleja en la jerarquía.

En el caso de las cadenas de valor modulares, los gruesos flujos de información se reducen a una entrega codificada en el enlace entre empresas, dejando a cada socio gestionar información tácita dentro de su propios límites firmes, o tal vez combinando alguna otra forma de gobierno de la cadena de valor, como cautivo o basado en el mercado, para parte de la cadena. Mientras que las relaciones entre los proveedores relacionales y modulares y las empresas que proporcionan sus insumos y componentes materiales se muestran como basadas en el mercado en la figura, igualmente podrían tomar otras formas. (Gereffi et al, 2005)

Figura 1. Tipología de la gobernanza de la cadena de valor.

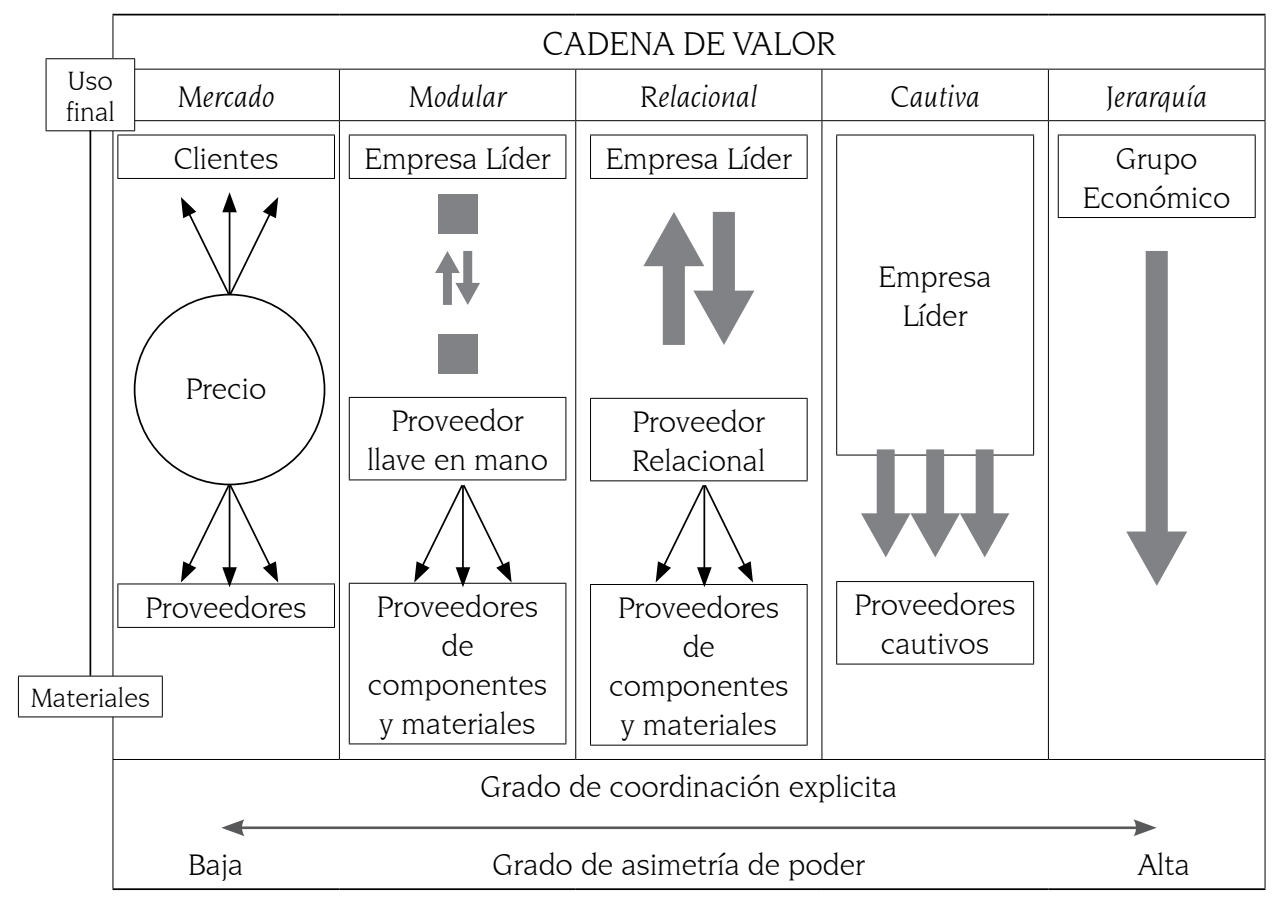

Fuente: Gereffi et al, (2005) y Gereffi (2014). 


\section{METODOLOGÍA}

Para el análisis del estilo de gobernanzas que ejercen las empresas líderes de la cadena de valor de la Bioenergía del Valle del Cauca, como se mencionó en la sección introductoria, se seleccionaron cinco empresas relevantes de las 2.891 empresas que conforman la cadena (Cámara de Comercio de Cali, 2015); se analizaron los informes de sostenibilidad de los años 2016 al 2019, y la información de documentos institucionales y gremiales, que aportaron datos sobre los indicadores de sostenibilidad y el relacionamiento con los proveedores, especialmente los de primera línea. Los criterios de selección de la empresa líder fueron: 1. Tamaño, 2. Sector al que pertenece, 3. Que promueva políticas de sostenibilidad, 4. Que elabore informes de sostenibilidad, 5. Que utilice indicadores de sostenibilidad como: GRI, Dow Jones Sustainability Index, CEPAL u otra reconocida.

En la cadena de valor se contemplan negocio como: cogeneradoras, generadores de biomasa, maquinaria agrícola, transporte y logística, semillas y fertilizantes, servicios de ingeniería, equipos eléctricos, distribuidoras y comercializadoras de energía y gas y destiladores (Camara de Comercio de Cali, 2014) Es importante considerar que el esfuerzo de pequeños agricultores dentro una cadena agroindustrial, se convierte en parte fundamental para su permanencia en el tiempo, de tal forma que tanto clientes como proveedores puedan satisfacer las necesidades que se traduzcan en una mejora de los aspectos social, económico y ambiental (Barrientos Felipa, 2015). En este sentido, una característica especial de la cadena de la bioenergía es la significativa representación de pequeños cultivadores de caña de azúcar.

Los proveedores más relevantes de la cadena se categorizan así:

En primer lugar estan los cultivadores y corteros de caña, que representan el $83 \%$ del total de los proveedores; en porcentaje menor esta el Sector financiero para efectos de apalancamiento; Empresas transportadoras que prestan servicios de transporte especializado de insumo y productos, y desplazamiento de personal; Industrias de Plásticos y Empaques; Fertilizantes y Abonos para el desarrollo de su actividad (Arango, Yoshioka y Gutiérrez, 2011); Productos de refinación y petróleo; Tecnología y Comunicaciones especializada, innovación con factor competitivo; Maquinaria Agrícola, para todo el proceso de cultivo, cosecha, transformación y producción (Asociación del Cultivadores de Caña de Azúcar de Colombia, 2020); Equipos eléctricos, Ingenios azucareros; Empresas forestales; Avícolas; Destiladora y Cogeneradores de energía, distribuidoreas y comercializadoras. (Cámara de Comercio de Cali, 2015)

Las empresas - en su mayoría pymes - que conforman los segmentos de negocio como: maquinaría agrícola, transporte y logística, semillas y fertilizantes, servicios de ingeniería y equipos eléctricos no cumplen con todos los criterios 
de selección establecidos para el estudio. Por su parte, las grandes empresas no cumplen con los requisitos 4 y 5 .

En Colombia, Valle del Cauca, la producción de la biomasa (bagazo de la caña de azúcar), materia prima principal para la cogeneración de energía eléctrica, es aportada por los ingenios azucareros, por lo tanto, se convierten en sus proveedores primordiales. En este sentido, de los nueve ingenios pertenecientes a cluster, cuatro de ellos no cumplen con el requisito 5; por tanto de los cinco ingenios que cumplen con los criterios de selección, se eligeron los que mayor incidencia han tenido en el sector bioenergético, incursionando en dos segmentos de la cadena como cogeneradores y destiladores, estos son: Grupo Agroindustrial Riopaila Castilla e Ingenio Manuelita S.A.; del sector papel está Carvajal Pulpa y Papel (Cogeneradora); del sector Forestal, Smurfit Kappa Cartón de Colombia S.A. (Cogenerador); del sector Energía y gas, Gases de Occidente (comercializador). Las tres últimas empresas se seleccionaron dado que son las únicas del sector que cumplen con los requisitos.

\section{RESULTADOS.}

\subsection{Grupo Agroindustrial Riopaila Castilla}

Grupo agroindustrial colombiano reconocido en el sector azucarero y bioenergético con experiencia en el mercado nacional e internacional, dedicado al cultivo de caña y sus derivados como: azúcar, alcohol carburante y energía eléctrica; también al cultivo de palma africana y producción de aceite crudo de palma. La sede administrativa se encuentra en Santiago de Cali, departamento del Valle del Cauca, y las sedes agroindustriales están ubicadas estratégicamente en los departamentos del Valle del Cauca, Vichada y Meta.

Riopaila Castilla S.A. es la compañía controlante o matriz del grupo empresarial formado para atender líneas de negocios a través de sus subordinadas; las filiales por las cuales se manejan unidades de negocios son: Destilería Riopaila S.A.S.; Riopaila Energía S.A.S.; Castilla Cosecha S.A.; Cosecha del valle S.A.S.; Riopaila Palma S.A.S.; Agroindustriales la Conquista S.A.S.; Asturias Holding SÁRL, cuenta con 5 sociedades de valores extrajeras y 29 compañías agroforestales S.A.S. (Grupo AgroIndustrial Riopaila Castilla, 2017). Es importante resaltar que el grupo empresarial a través de sus filiales maneja diferentes actividades de la cadena como: Destileria Riopaila S.A.S., Riopaila Castilla S.A. y Riopaila Energía S.A.S. lo que hace que el grupo sea proveedor y comprador. 
El grupo Riopaila Castilla cuenta con un código de mejores prácticas corporativas en donde se estipula los siguientes comités: Auditoría, Gobierno Corporativo, Gestión Humana y Responsabilidad Social Empresarial y Ética. De igual forma tienen constituido dos comités de sostenibilidad: el comité estratégico de sostenibilidad, a cargo de la alta dirección y presidido por el presidente de la compañía, y el comité técnico de sostenibilidad, este último implementa las políticas y dirige el equipo técnico de sostenibilidad. Ambos se rigen por los valores y principios de sostenibilidad: honestidad, compromiso, lealtad y respeto.

En su relacionamiento con los proveedores, Riopaila Castilla diferencia dos grupos de proveedores: el primero, con quien mantiene una fuerte relación, son los proveedores de caña y tierras (alquilan la tierra o venden la caña); el segundo suministra bienes materiales, repuestos, insumos de campo, entre otros. Con los primeros, tienen actividades especiales como: grupos de trasferencia tecnológica, en donde capacitan a los cañicultores en temas de actualidad y tecnología referente al agro reafirmando su compromiso con la sostenibilidad; visitas personalizadas para atender diferentes inquietudes; día de campo con proveedores, este encuentro anual de proveedores de caña busca fortalecer el relacionamiento con sus aliados estratégicos, un espacio dedicado a dar a conocer acciones estratégicas que conducen a la contribución del desarrollo de la región.

Mediante el programa Oferta Diferenciadora que se sustenta en tres estrategias COE, cercanía, oportunidad y ejecución, el grupo Agroindustrial procura el mejoramiento continuo de la calidad, oportunidad en los servicios y labores agrícolas que se le prestan a los cañicultores: El acercamiento a los proveedores de caña permite que ellos identifiquen sus necesidades y expectativas (Grupo Agroindustrial Riopaila Castilla, 2019).

Con relación a los proveedores de bienes, obras y servicios, con el apoyo de la fundación Caicedo González - Riopaila Catilla, desarrollan proyectos para mejorar la productividad y competitividad de las mypimes proveedoras, perteneciente a los diferentes eslabones de la cadena productiva, entre las actividades de los proyectos están: Implementación de buenas prácticas y sistemas de gestión de calidad, certificación bajo estándares internacionales de Gestión de Calidad, Implementación del Sistema de Gestión Ambiental, Implementación de los requisitos de Salud y Seguridad en el Trabajo, Fortalecimiento de la gestión financiera y costos, Acompañamiento técnico especializado en Gestión Comercial, Acompañamiento técnico en DDHH bajo parámetros del Pacto Global y GRI, dando cumplimiento a los requisitos de Riopaila Castilla S.A., PACTO GLOBAL, GRI y Ministerio del Trabajo. (Grupo Agroindustrial Riopaila Castilla, 2017) 
Por otro lado, Riopaila Castilla realiza auditoria de cumplimiento a sus proveedores para determinar si cumplen con los criterios de sostenibilidad propuestos por ellos. De igual forma a los proveedores nuevos le realizan evaluación y selección de acuerdo a criterios de sostenibilidad (Grupo Agroindustrial Riopaila Castilla, 2019).

\subsection{Ingenio Manuelita S.A.}

Manuelita S.A., es otra de las grandes empresas del sector agroindustrial del Valle del Cauca, constituida en 1864, fue el primer ingenio en Colombia, con posición en el mercado, dentro de las actividades se destacan: el azúcar, energía, aceites de palma, Mejillones, frutas y hortalizas. Manuelita cuentas con sede en Colombia, Perú, Chile y Brasil (Manuelita S.A., 2019).

La organización identifica ocho prioridades de sostenibilidad como objetivos estratégicos, cada prioridad está enmarcada en los tres pilares de la sostenibilidad y a su vez, cumplen con los ODS de Naciones Unidas. Dentro de las prioridades económicas está el desarrollo de proveedores competitivos y sostenibles, otro aspecto de relevancia en la organización (Manuelita S.A., 2019).

El ingenio Manuelita, considera a sus proveedores aliados estratégicos para el desarrollo y sostenibilidad del negocio, cerca del $85 \%$ de las compras, las realizan en el área loca. Dentro de los proyectos que tienen para mejorar las condiciones productivas y rentables de los proveedores, de acuerdo a la información obtenida de los Informes de sostenibilidad de Manuelita S.A. (2019), se encuentran:

Asesoría Técnica y Actualización: la organización dispone de un área de tecnología agrícola encargada de investigación y programas de transferencia de tecnología en labores de cultivo dirigidos a proveedores de materia prima agrícola.

Grupos de transferencia de tecnología-GIT: reunión bimensual con proveedores para socializar temas relacionados con variedades de producción, métodos de riego y otros temas de importancia, con el propósito de aumentar la productividad y disminuir el impacto ambiental. Desarrollos Propios: cuando realizan un desarrollo tecnológico propio, capacitan a sus proveedores con el fin de ponerlo en marcha. Días de Campo: dos días al año se celebra una reunión con los proveedores en la que participa la autoridad ambiental para promover el mutuo acercamiento, compartir buenas prácticas. Satisfacción de Proveedores: la satisfacción de proveedores se mide por una encuesta elaborada por un externo, la cual trabaja con base en objetivos estratégicos como: proveedores competitivos y sostenibles, suministro de la materia prima y bienestar a la comunidad. (Castro y Moreno, 2015)

Manuelita S.A. realiza Auditoría Técnica Participativa, que consiste en la revisión permanente el área del campo para constatar el cumplimiento de las disposiciones 
normativas. Al igual que Riopaila cuenta entre las líneas de negocio, la destilería y la cogeneración de energía, que también lo hace proveedor y comprador de la cadena.

Es importante mencionar que los ingenios tienen tres tipos de contratos ${ }^{2}$ con los proveedores de caña de azúcar, donde se estipula las condiciones, el valor y forma de pago.

El cañicultor decide qué variedad de caña va a sembrar; también se evidencia ventas de semilleros de los ingenios a los cañicultores. Los ingenios, en su interés de mejorar la calidad y la productividad de los cultivos, se convierten en clientes y proveedores de los cañicultores. (Arango Sanclemente et al, 2011)

\subsection{Carvajal Pulpa y Papel S.A.}

El grupo Carvajal se creó en 1904 en la ciudad de Cali, Colombia, como una empresa familiar, en la actualidad es una organización multilatina con presencia en 14 países de América, con más 19.000 colaboradores, atendiendo diferentes sectores empresariales como: Empaque, Propal, Educación, Tecnología y Servicios, Mepal y Soluciones de Comunicaciones.

Las empresas que conforman el grupo empresarial Carvajal se rigen por las políticas y lineamientos del gobierno corporativo, de igual forma los grupos de interés, por tanto, la organización expresa que el relacionamiento con los proveedores, "está regida por los lineamientos éticos y políticas que bridan un marco de referencia conductual para sus grupos de interés, en los que puedan actuar dentro de los estándares esperados en términos de ética" (Carvajal S.A., 2019).

Los proveedores deben seguir las normas mientras dure el vínculo comercial; la organización Carvajal, considera a este grupo de interés como socios en la satisfacción de las necesidades de los clientes y por tanto las decisiones de compras se basan en la confiabilidad, eficiencia, calidad, servicio y costo, buscando siempre el mutuo beneficio (Carvajal S.A., 2019). Por otro lado, expresan que dan la posibilidad a proveedores locales de las comunidades, donde operan participar en proyectos conjuntos.

En este sentido, Carvajal en su relacionamiento con los proveedores tiene reglas claras de estricto cumplimiento, que son comunicadas y socializadas a través de la página web del grupo empresarial.

Lineamiento éticos y políticas a seguir por los proveedores:

2 Contrato de Arrendamiento de la finca; contrato cuenta en participación y contrato caña en mata (el proveedor productivo) (Castro y Moreno, 2015). 
A. Derechos humanos y laborales: Rechazar el trabajo infantil y trabajo forzoso; respetar la dignidad de las personas, evitando la discriminación por razón de discapacidad, origen étnico, religión, orientación sexual, edad, sexo, afinidad política, estado civil o ciudadanía; Respetar la libertad de asociación y negociación colectiva; Garantizar que los colaboradores cuenten con un régimen laboral que reúna el conjunto de normas y condiciones conforme a las cuales se debe regir; Generar un ambiente de trabajo seguro, digno y saludable, cumpliendo con la normatividad legal en materia de seguridad y salud en el trabajo. B. Sistema de prevención de lavado de activos y Financiación del terrorismo (Siplaft): Cumplimiento de Políticas de anticorrupción y antisoborno transaccional. C. Manual de cumplimiento, los proveedores deben contar con políticas anticorrupción y antisoborno transaccional y actuar dando estricto cumplimiento. D. Competencia. se prohíbe los comportamientos anti-competitivos y abusos de la posición dominante del mercado.

E. Manejo de datos personales, el adecuado uso y tratamiento responsable de los datos personales de los colaboradores y terceros. F. Instructivo de seguridad y salud en el trabajo, políticas a las que se compromete el proveedor en la identificación del riesgo a los que se expondrán las personas que trabajan para ellos, capacitación en la prevención del riesgo y la implementación de SG_SST. 7. Medio ambiente, las actividades de producción deben desarrollarse dentro del marco de la protección del medio ambiente, fortaleciendo el desarrollo sostenible y la producción limpia. (Carvajal S.A, s.f)

Por su parte, Carvajal Pulpa y Papel se rige por las políticas del grupo Carvajal, cuenta con dos plantas de producción en Guachené y Yumbo, regiones azucareras del Valle del Cauca, donde se ubican los ingenios azucareros, proveedores del bagazo de la caña de azúcar, materia prima para la producción del papel y para la cogeneración de energía.

Cabe mencionar que en el Valle del Cauca se procesan cerca de 24 millones toneladas de caña de azúcar al año, para lo cual el bagazo de la caña representa el $30 \%$ de la caña molida, de este porcentaje el $85 \%$ es utilizado por los ingenios azucareros para la cogeneración de energía y el 15 \% es utilizado por Carvajal Pulpa y Papel (Becerra et al, 2016).

\subsection{Gases de Occidente S.A. E.S.P.}

La empresa Gases de Occidentes S.A., se dedica a la comercialización de gas natural, aportar soluciones energéticas y servicios complementarios, en los departamentos del Valle del Cauca y norte del Cauca.

Para esta organización, el relacionamiento con los grupos de interés es un punto determinante para tener una gestión sostenible y generar valor agregado a los mismos. En el informe de sostenibilidad, expresan que las actuaciones están centradas en el ser humano como eje fundamental sustentadas en la ética y la 
transparencia (Gases de Occidente, 2018, p.19). La entidad cuenta con un comité de sostenibilidad con responsabilidad en asuntos económicos, ambiental y social encargados de hacer seguimiento a los planes y proyectos relacionados con la sostenibilidad y el relacionamiento con los grupos de interés.

En relación con los derechos humanos, los respetan y valoran la diversidad, derecho innegable e inherente a todas las personas, las cuales deben recibir un trato igualitario. Por tanto, en los contratos firmados por la empresa se tiene una cláusula sobre cumplimientos de las disposiciones en derechos humanos que los proveedores y contratistas se comprometen a cumplir dentro del marco de la legalidad colombiana, que garanticen el respeto de los derechos humanos en su empresa (Gases de Occidente, 2018, p. 26).

En cuanto al relacionamiemto con los proveedores, los contratistas se encuentran en el marco del cumplimiento de los lineamientos de seguridad, salud y medio ambiente definidos por la empresa. Para la verificación del cumplimiento realizan auditoria de seguimiento a las empresas y a las obras ejecutadas. En el mismo sentido desarrollan actividades para el fortalecimiento de la seguridad de las empresas contratistas como: capacitanción en seguridad y salud ocupacional a sus empleados, capacitación al personal operativo encargado de los sistemas medio ambientales, reuniones tecnicas, desarrollo de comites HSE y comité gestión con contratistas donde se divulgan los resultados de gestión de cada periodo (Gases de Occidente, 2018).

\subsection{Smurfit Kappa Cartón de Colombia S.A.}

Cartón Colombia S.A es una organización dedicada a la fabricación de papel y productos de empaques, cuyos segmentos de operación son: pulpa, papel y cartón, empaques y reciclaje. Cuenta con la división forestal y una división de reciclaje.

Comercialmente se conoce como Smurfit Kappa grupo económico reconocido mundialmente como productor de papel (Smurfit Kappa Colombia, 2016), su principal materia prima proviene de las plantaciones propias de eucalipto y pino; y sus principales proveedores son las fincas productoras de árboles de las fincas y los ingenios azucareros.

Murfit Kappa realiza su actividad empresarial con un alto nivel de ética. Provee una guia de responsabilidades ética y legales que deben cumplir los grupos de interes (Smurfit-Kappa, s.f), en este mismo sentido son tomados en cuenta los Objetivo para el Desarrollo Sostenible de las Naciones Unidas, con prioridades materiales en toda la cadena de valor (Smurfit Kappa, 2018). 
Las operaciones del grupo se centran en un modelo de economía circular, sistema en el que la productividad de los recursos que se utilizan lo maximizan y los residuos generados a través de sus productos se minimizan, reutilizando los recursos que extraen, como los residuos forestales utilizados para la generación de energía. La filosofía de circuito cerrado empieza en las plantaciones forestales, garantizando que el $100 \%$ de la fibra virgen para producir papel se obtiene de plantaciones forestales manejadas responsablemente donde los derechos humanos y la biodiversidad son primordiales; los residuos forestales se utilizan en la generación de energía. (Smurfit Kappa, 2017)

El modelo circular de negocio involucra a los grupos de interés, compartiendo conocimiento, experiencia y experticia, permitiendo fortalecer la sostenibilidad en la cadena de valor.

De igual forma las actividades que adelanta con los proveedores son: en temas de innovación, realizan acompañamiento en pruebas y optimización en innovación de productos y procesos; abastecimiento de productos y servicios locales, ayudando a dinamizar las economías de las regiones. Los proveedores de primer nivel son los ingenios azucareros, las fincas productoras de árboles y transportes de carga.

\subsection{Tipología de la gobernanza en la cadena de valor de la bioenergía en el valle del cauca}

De acuerdo al modelo planteado por Gereffit et al (2005) y Gereffi (2014), se analizan las relaciones de las empresas líderes de la cadena con sus proveedores. En la tabla 2 se evidencia el tipo de gobernanza que se tiene principalmente con los proveedores de primera base.

Para el caso del Grupo Agroindustrial Riopaila Castilla y el Ingenio Manuelita, predomina el tipo cautiva con los proveedores de la caña de azúcar, dado que la relación entre cañicultores y los ingenios es estrecha, directa y circular, debido al carácter perecedero de la caña y la imposibilidad de transarla internacionalmente, los únicos demandantes de caña de azúcar son los ingenios (Arango et al, 2011), de acuerdo al análisis realizado, los ingenios ejercen un acompañamiento, monitoreo y auditoria a los proveedores de la caña de azúcar; en el caso específico de Riopaila Castilla también se da una relación de Jerarquía ya que esta provee de Biomasa a Destilería Riopaila S.A.S. y a Riopaila Energía S.A.S., filiales de grupo económico, por tanto el grado de coordinación explicita y asimetría de potencia de poder es alta (Ver tabla 1). En el caso de los proveedores de maquinaría agricola y fertilizantes la relación está dada más por el precio del mercado; por tanto, el costo de cambiar de proveedor es bajo. En el caso del transporte especializado, llamados "trenes cañeros", los proveedores elaboran los productos o servicios 
según los requerimientos de los ingenios, configurandose así una tipología modular. Con relación a la industria para la planta, existe una interacción compleja entre los ingenios y las empresas proveedoras, creando una mutua dependencía dada por la alta tecnología y la especificidad de la maquinaría utilizada en el sector azucarero y bioenergético.

Tabla 2. Tipo de gobernanza de la cadena de valor de la bioenergía

\begin{tabular}{|c|c|c|c|c|c|}
\hline $\begin{array}{l}\text { Empresa líder } \\
\text { Tipo de } \\
\text { Gobernanza }\end{array}$ & $\begin{array}{c}\text { Grupo } \\
\text { Agroindustrial } \\
\text { Rio Paila } \\
\text { Castilla } \\
\end{array}$ & Manuelita S.A. & $\begin{array}{l}\text { Carvajal S.A. } \\
\text { Pulpa y Papel }\end{array}$ & $\begin{array}{l}\text { Gases de } \\
\text { Occidente }\end{array}$ & $\begin{array}{l}\text { Smurfit Kappa } \\
\text { Cartón de } \\
\text { Colombia S.A. }\end{array}$ \\
\hline Mercado & $\begin{array}{l}\text { Proveedores } \\
\text { de maquinaria } \\
\text { agrícola, } \\
\text { fertilizantes y } \\
\text { otros. }\end{array}$ & $\begin{array}{l}\text { Proveedores } \\
\text { de } \\
\text { maquinaria } \\
\text { agrícola, } \\
\text { fertilizantes y } \\
\text { otros. }\end{array}$ & $\begin{array}{l}\text { Proveedores } \\
\text { de bienes y } \\
\text { servicios no } \\
\text { especializados. }\end{array}$ & $\begin{array}{c}\text { Proveedores } \\
\text { de bienes y } \\
\text { servicios no } \\
\text { especializados. }\end{array}$ & $\begin{array}{c}\text { Proveedores } \\
\text { de bienes y } \\
\text { servicios no } \\
\text { especializados. }\end{array}$ \\
\hline Modular & $\begin{array}{c}\text { Transporte } \\
\text { especializado. }\end{array}$ & $\begin{array}{c}\text { Transporte } \\
\text { especializado. }\end{array}$ & $\begin{array}{l}\text { Proveedores de } \\
\text { Biomasa. } \\
\text { Ingenios } \\
\text { azucareros. }\end{array}$ & & $\begin{array}{l}\text { Proveedores } \\
\text { de Biomasa. } \\
\text { Ingenios } \\
\text { azucareros. }\end{array}$ \\
\hline Relacional & $\begin{array}{l}\text { Industria para } \\
\text { la planta de } \\
\text { producción. }\end{array}$ & $\begin{array}{l}\text { Industria para } \\
\text { la planta de } \\
\text { producción. }\end{array}$ & $\begin{array}{l}\text { Industria para } \\
\text { la planta de } \\
\text { producción. }\end{array}$ & $\begin{array}{c}\text { Proveedores } \\
\text { de bioenergía } \\
\text { - ingenios } \\
\text { azucarero. }\end{array}$ & $\begin{array}{l}\text { Industria para } \\
\text { la planta de } \\
\text { producción. }\end{array}$ \\
\hline Cautiva & $\begin{array}{l}\text { Proveedores } \\
\text { de caña de } \\
\text { azúcar - } \\
\text { bagazo de la } \\
\text { caña. }\end{array}$ & $\begin{array}{l}\text { Proveedores } \\
\text { de caña de } \\
\text { azúcar - } \\
\text { bagazo de la } \\
\text { caña. }\end{array}$ & N.A. & Contratistas. & $\begin{array}{l}\text { Fincas } \\
\text { productoras de } \\
\text { madera. }\end{array}$ \\
\hline Jerarquía & $\begin{array}{c}\text { Para la } \\
\text { generación } \\
\text { se tiene } \\
\text { destilería } \\
\text { Riopaila S.A.S. } \\
\text { y Energía } \\
\text { S.A.S. (filial). }\end{array}$ & N.A. & N.A. & N.A. & $\begin{array}{l}\text { Proveeduría } \\
\text { de madera } \\
\text { de pino y } \\
\text { eucalipto. }\end{array}$ \\
\hline
\end{tabular}

Fuente: elaboración propia, 2020.

En el caso de Carvajal Pulpa y Papel S.A. los proveedores principales de la materia prima para la elaboración del papel y la energía, son los ingenios azucareros, esta relación se caracteriza porque tanto el proveedor como el comprador, tienen 
capacidades elevadas, el proveedor entrega la materia prima de acuerdo a las especificidades del cliente, la asimetría de poder entre ambos es baja, el costo de cambiar de proveedor es igualmente bajo, por tal motivo, se dice que es de tipo modular; los proveedores de bienes y servicios no especializados son de tipo mercado.

Por su parte, Gases de Occidente es el principal proveedor de Bioenergía son los ingenios azucareros, pero a diferencia de Carvajal Pulpa y Papel S.A. la relación cambia ya que la especificación del producto es alta, las transacciones son complejas y el conocimiento tácito que deben intercambiarse es relativamente alto, se puede decir que la dependencia es mutua, aunque los ingenios utilizan la energía principalmente para consumo propio y en un porcentaje bajo la comercializan. Por las características anteriores existe un tipo relacional.

Con referencia a Smurfit Kappa Cartón de Colombia S.A. ellos cuentan con plantaciones propias de pino y eucalipto que permiten el autoabastecimiento de la materia prima para la fabricación del papel, también se abastecen de fincas productoras de madera y de un ingenio azucarero. Con las fincas productoras se tiene una relación de tipo cautiva dado el trato cercano y el monitoreo que ejerce en las fincas proveedoras y con el ingenio la relación es de tipo modular.

En cuanto a proveedores de industria para planta de producción, por ser plantas con altas especificaciones, para las cinco compañías seleccionadas el tipo de gobernanza es la relacional.

Con referencia a los proveedores de repuestos y fabricante de pasadores, rodillos, arañas y otras piezas para la utilización en el proceso productivo de la caña, reparación de trenes cañeros, las relaciones entre las empresas de maquinaria agrícola y los cultivadores de caña de azúcar permiten que los segundos encuentren una amplia oferta de este tipo de productos para satisfacer sus necesidades (Arango et al, 2011), dándose en este caso una relación de tipo mercado.

\section{CONCLUSIONES}

Este trabajo contribuye al avance en el estado del arte del estudio de las tipologías de gobernanza de las cadenas de valor, al aplicar los criterios de los trabajos seminales de Gereffit et al (2005) y Gereffi (2014), al sector de la bioenergía del Valle del Cauca; para de esta manera determinar la posibilidad de transferencia de políticas de sostenibilidad de la empresa líder a sus proveedores, puesto que, los requerimientos que las empresas líderes hacen estos últimos, en materia de buenas prácticas en las dimensiones ambiental, social y económica, se constituye en una alternativa para 
que la cadena de valor opere de manera sostenible, lo que también se traduce en mejora en la competitividad.

Al tratarse de un estudio de casos no se puede hablar de representación estadística. No obstante, al tratarse de cinco empresas líderes del sector, si puede decirse que es relevante y, por tanto, sirve como base para futuros trabajos de corte cuantitativo en el sector de la bioenergía del Valle del Cauca.

En la cadena de valor de la bioenergía predominan el tipo de gobernanza cautiva y modular, facilitando esta tipología en la adopción de políticas de sostenibilidad por parte de los proveedores. Con respecto a la relación que se da entre proveedores y compradores con capacidad elevadas, se evidenció que estas empresas cuentan con políticas de sostenibilidad claramente definidas y estas se encargan de difundirla a sus proveedores.

Futuros estudios podrán ser de corte cuantitativo y emplear métodos de ecuaciones estructurales que lleven a definir las variables independientes, mediadoras e independientes y a establecer su incidencia en el funcionamiento de la cadena de valor del sector de la bioenergía del Valle del Cauca como una cadena de valor sostenible.

El sector económico más importante del Valle del Cauca, Colombia, es el agroindustrial, principalmente el sector azucarero, cogenerador y mayor proveedor de la materia prima de la bionenergia (bagazo de la caña de azucar); ante la posibilidad de que las empresas lideres peermen a sus proveedores las politicas de sostenibilidad, redunda en la calidad y competitividad del sector bioenergético y por ende contribuye al desarrollo economómico de la región, a mejorar la calidad de vida de los habitantes y contribuye con la preservación del medioambiente.

Las empresas que componen el sector agroindustrial azúcarero y que a su vez conforman el cluster de la bionergía, se han visto afectados por la pandemia COVID-19, a razón de que en la producción del azúcar, producto de la canasta básica familiar, se genera el bagazo de la caña del que se produce el etanol, utilizado en gran cantidad para el biocombustible y por tanto debido al confinamiento se produjeron sobreinventarios de etanol dado la reducción del consumo de gasolina; como medida de protección a este sector el Gobierno Nacional para mitigar el impacto económico decretó: que "como consecuencia de la enfermedad del coronavirus, requiere adoptar medidas para restringir la importación de bienes como etanol, para prevenir el colapso de una industria de interés estratégico para la nación como es la azucarera" (Decreto 527 de 2020). 
En este sentido el sector se afectó parcialmente en la parte económica, pero se vio fortalecido en la dimensión social ya que las diferentes empresas mostraron la solidariadad con sus proveedores, con los trabajadores y con la comunidad en general, donando más de un millón de kilos de azúcar y más de un millón de litros de etanol (alcohol) para enfrentar y prevenir la COVID-19; por su lado, las empresas comercializadoras de la bioenergía, adelantaron programas para proteger a los consumidores como diferir los cobros de energía, dar plazos amplios, no realizar cortes de servicios públicos para los consumidores morosos, entre otros beneficios.

\section{BIBLIOGRAFÍA}

Arango Sanclemente, S., Yoshioka Vargas, A. M., y Gutiérrez Rincón V. (2011). Analisis de ambiente competitivo del cluster agroindustrial del azúcar en el valle geográfico del azúcar. Desarrollo de retos. Pontificia Universidad Javeriana Cali. http://vitela.javerianacali.edu.co/bitstream/ handle/1 1522/3452/LibroCluster.pdf?sequence $=1$ EisAllowed $=\mathrm{y}$

Asociación de Cultivadores de Caña de Azucar de Colombia (2020). Informe de Sostenibilidad 2019 - 2020. ASOCAÑA ORG., https://www.asocana.org/documentos/2972020-1255B6DD-00FF00,000A000,878787,C3C3C3,0F0F0F,B4B4B4,FF00FF,2D2D2D,A3CAB4.pdf

Barrientos Felipa, P. (2015). La cadena de valor del cacao en Perú y su oportunidad en el mercado mundial. Semestre Económico, 18(37), 129-156. https://doi.org/10.22395/seec.v18n37a5

Becerra, A. P., Buitrago, A. L., y Pinto, P. (2016). Sostenibilidad del Aprovechamiento del Bagazo de la Caña de Azúcar, en el Valle del Cauca, Colombia. Ingeniería Solidaria, 12(20),133-149. http://dx.doi.org/10.16925/in.v12i20.1548

Camara de Comercio de Cali. (2014). Bionergía . Recuperado el Julio de 2020, de Sitio Web de la Camara de Comercio de Cali: https://www.ccc.org.co/revista-accion-ccc/bioenergia/

Cámara de Comercio de Cali. (2015). Bioenergía. Editorial construcción colectiva]. Revista acción digital. Edición 164. http://www.ccc.org.co/revista-accion-ccc/bioenergia/

Castro A. M., y Moreno C. G. (2015, 17-19 de septiembre). La incidencia de las Empresas líderes de la cadena de valor agroindustrial del sector azucarero en la adopción de criterios de sostenibilidad [ponencia]. 4to Simposio Internacional de Investigación en Ciencias Económicas, Administrativas y Contables - Sociedad y Desarrollo. Bogotá, Colombia. http://www.unilibre. edu.co/bogota/pdfs/2016/4sin/B61.pdf.

Carvajal (2019). Nuestro Actuar Ético. https://www.carvajal.com/wp-content/uploads/2016/10/ NuestroActuarEtico.pdf.

Carvajal S.A. (s.f). Proveedores. https://www.carvajal.com/index.php/nuestra-empresa/proveedores/

Gases de Occidente (2018). Informe de Gestión Sostenible 2018-GdO Gases de Occidente. Gases de Occidente.

Gereffi, G., Humphrey, J., y Sturgeon, T. (2005). The Governance of Global Value Chains. Review of International Political Economy, 12(1), 78-104. http://www.jstor.org/stable/25124009. 
Gereffi, G. (2014). A global value chain perspective on industrial policy and development in emerging markets. Duke Journal of Comparative E International Law, 24, 433-458. https://scholarship. law.duke.edu/cgi/viewcontent.cgi?article $=1404 \&$ conte $=$ djcil

Grupo Agroindustrial Riopaila Castilla. (2017). Informe de sostenabilidad y gestión. Grupo Agroindustrial Riopaila Castilla.

Grupo Agroindustrial Riopaila Castilla (2019). Informe de sostenibilidad y gestión. Grupo Agroindustrial Riopaila Castilla.

Kardos, M. (2012). The reflection of good governance in sustainable development strategies. Procedia - Social and Behavioral Sciences, 58(12), 1166-1173. https://doi.org/10.1016/j. sbspro.2012.09.1098

Humphrey, J., y Schmitz, H. (2002). How Does Insertion in Global Value Chains Affect Upgrading in Industrial Clusters? Regional Studies, 36(9), 1017-1027. DOI: 10.1080/0034340022000022198

Manuelita S.A. (2019). Informe de Sostenibilidad 2017-2018. Manuelita S.A.

Pérez Ibáñez, J. (2019). Cadenas Globales de Valor. Una Revisión bibliográfica. Semestre Económico, 22(51) 63-81. https://doi.org/10.22395/seec.v22n51a4

Presidencia de la Republica (2020, 7 de abril). Decreto No. 527. Por el cual se regula el exceso de inventarios del alcohol carburante para prevenir el colapso de la producción del azúcar en el marco de la emergencía económica social y ecológica. https://dapre.presidencia.gov.co/normativa/normativa/ DECRETO\%20527\%20DEL\%207\%20DE\%20ABRIL\%20DE\%202020.pdf

Santarcángelo, J., Schteingart, D., y Porta, F. (2017). Cadenas Globales de Valor: una mirada crítica a una nueva forma de pensar el desarrollo. CEC, 7, 99-129. http://www.unsam.edu. ar/escuelas/economia/revistaedd/wpcontent/uploads/2017/11.pdf

Seuring, S., y Müller, M. (2008). From a literature review to a conceptual framework for sustainable. Journal of Cleaner Production, 16(15), 1699-1710. https://doi.org/10.1016/j.jclepro.2008.04.020

Smurfit Kappa (s.f). Código de Conducta. Smurfit Kappa Carton Colombia. http://www. smurfitkappa.com

Smurfit Kappa (2017). Informe de Sostenibilidad 2017. http://www.smurtifkappa.com

Smurfit Kappa (2018). Sostenibilidad en cada fibra. Smurfit Kappa.

Smurfit Kappa Colombia (2016). 70 años de travesia hacia el futuro. Villegas Editores.

Stancey, F., E Garay, G. (2010). La gobernabilidad en la cadena de valor. USAID. From tha America People. Informe. https://www.microlinks.org/node/2261

Sturgeon, T. (2013). Global Value Chains and Economic Globalization. Eurostat or the Massachusetts Institute of Technology. Massachusetts: Eurostat's work on global value chains and international sourcing. ITM.

Tamayo, I., Gutierrez, L., y Ruiz , A. (2018). Boosting sustainability and financial performance: the role of supply chain controversies. International Journal of Production Research, 57(11), 1-17. https://doi.org/10.1080/00207543.2018.1562248 